\section{Siedlungsflächenentwicklung und Nutzungskonkurrenzen}

\author{
von Fabian Dosch, Bundesamt für Bauwe- \\ sen und Raumordnung ${ }^{1}$
}

\begin{abstract}
Basierend auf den regionalisierten Entwicklungstrends der Flächeninanspruchnahme (rückblickend - aktuell - künftig) skizziert der Beitrag Nutzungskonkurrenzen durch die Siedlungsexpansion in Deutschland. Neben Konkurrenzen mit Landwirtschaft, dem Boden-, Freiraumund Naturschutz sowie stadtwirtschaftlichen Kalkulationen werden auch Flächenbedarfe durch die Bereitstellung erneuerbarer Energien erörtert. Nach der Evaluierung der Zielerreichung nachhaltiger Siedlungsentwicklung erfolgt eine Übersicht zu Strategien zur Flächenentwicklung auf Bundesebene durch Forschung, Förderung und Rechtssetzung und zu perspektivischen Maßnahmen. Zentrale Stellgröße ist dabei das Flächenrecycling im Kontext einer Flächenkreislaufwirtschaft.
\end{abstract}

\section{Entwicklung und Trends der Flächeninanspruchnahme}

Ein sparsamer und schonender Umgang mit der begrenzten Ressource Fläche gehört zu den Kernelementen der nationalen Nachhaltigkeitsstrategie. Ein zentrales, weithin bekanntes und wiederholt bekräftigtes Ziel der Flächenhaushaltspolitik der Bundesregierung ist die Verminderung der Flächeninanspruchnahme für Siedlung und Verkehr auf 30 ha pro Tag im Jahr 2020 (Dosch 2007). Als der Rat für Nachhaltige Entwicklung die 21 indikatorenbasierten Ziele der Nachhaltigkeitsstrategie im April 2008 auf den Prüfstand stellte (RNE 2008), stand die Ampel für den Indikator Flächeninanspruchnahme auf Rot: Das Etappenziel wird deutlich nicht erreicht. Wurden im Jahr 2002 durchschnittlich 105 ha pro Tag in Anspruch genommen, waren es im Jahr 2005 wieder 118 ha pro Tag. Doch ist dem tatsächlich so?

\subsection{Flächenneuinanspruchnahme gebremst}

Nach der Statistik hat die Siedlungs- und Verkehrsfläche ${ }^{2}$ seit 1992 um insgesamt 6.133 $\mathrm{km}^{2}$ oder $15,2 \%$ weiter zugenommen, darunter die Siedlungsfläche mit $20,7 \%$ deutlich stärker als die Verkehrsfläche mit 7,2\%. Die jährliche „Flächenneuinanspruchnahme“ ist aber seit dem Jahr 2000 leicht rückläufig. Ein Grund ist der drastische Rückgang der Bautätigkeit der vergangenen Jahre - insbesondere beim Wohnungsbau. Seit dem Höhepunkt von 129 ha pro Tag im Zeitraum 1997 bis 2000 sank die Flächenneuinanspruchnahme für Siedlungs- und Verkehrszwecke inzwischen auf 113 ha pro Tag im Zeitraum 2003 bis 2006 (vgl. Abb. 1 nächste Seite).

Allerdings sind die statistischen Zunahmen der Siedlungs- und Verkehrsfläche durch Umstellungen in der Flächenstatistik insbesondere bei den Erholungsflächen deutlich überhöht. Nach Schätzungen dürfte die tatsächliche Neuinanspruchnahme im Zeitraum 2003 bis 2006 bei unter 95 ha pro Tag gelegen haben. Betrachtet man nur den Zuwachs an Gebäude- und Freiflächen, so hat er sich bundesweit von 82 ha (1993-1996) auf 43 ha pro Tag (2003-2006) fast halbiert und ist in den östlichen Bundesländern noch stärker rückläufig als im Westen. Entsprechend gingen in der letzten Dekade auch die Baufertigstellungen im Wohnungsbau um ca. zwei Drittel zurück. Im gewerblichen $\mathrm{Bau}$ - je nach Bezugsgröße, aber besonders in den neuen Bundesländern war die Abnahme sogar noch stärker (ZDB 2008; BBR 2006). Soll dies ohne Wirkung auf die Flächeninanspruchnahme gewesen sein? Gewiss nicht.

Die Probleme sind jedoch durch die verminderte, aber weiterhin anhaltende Flächenneuinanspruchnahme kaum kleiner geworden. Denn insbesondere die vermehrten brachliegenden Siedlungsflächen und der Leerstand im Siedlungsbestand stellen die Stadtentwicklung vielerorts vor neue Herausforderungen. 
Abb. 1： Tägliche Veränderung der Siedlungs- und Verkehrsfläche (1993-2006)

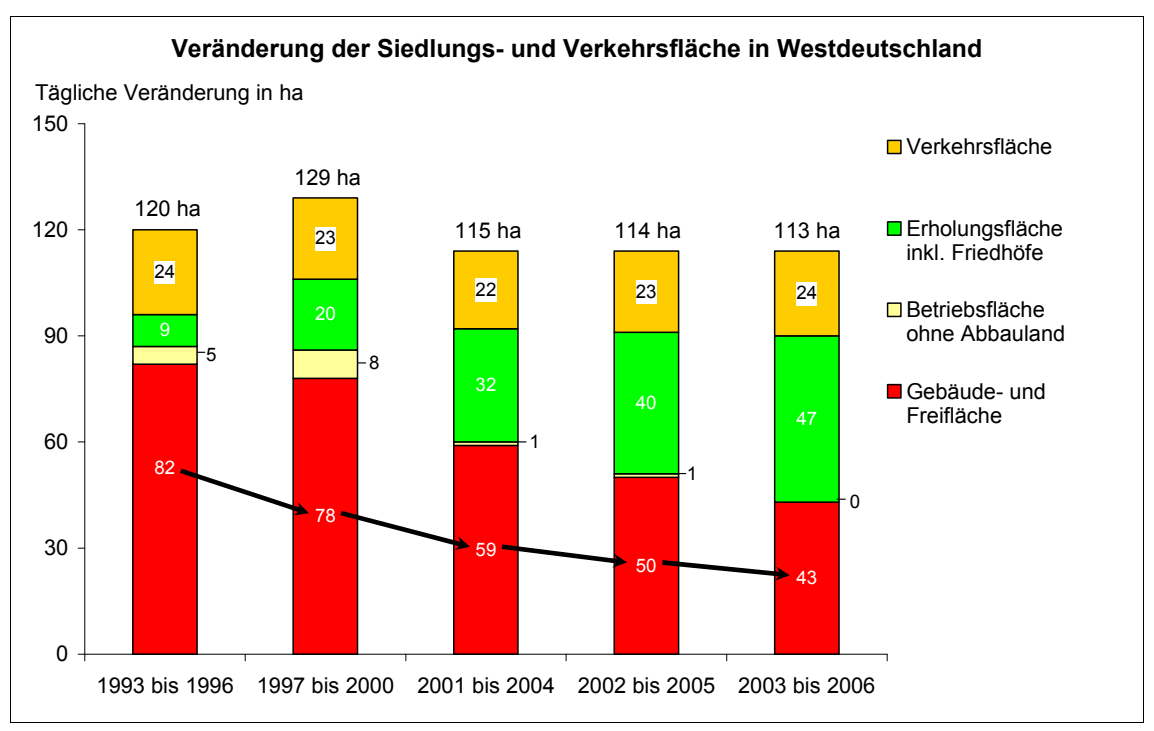

Quellen: Statistisches Bundesamt, eigene Berechnungen, (C BBR Bonn 2008

\subsection{Wohnbauflächen wuchsen deutlich stärker als Nichtwohnbauflächen}

Die Wohnungsbaufertigstellungen insgesamt sanken bundesweit bereits seit 1995 von 603.000 Wohnungen nahezu kontinuierlich auf 249.000 im Jahr 2006; dies entspricht einem Rückgang um 58,6\%. Andererseits stieg der Anteil der Ein- und Zweifamilienhäuser an der Errichtung neuer Wohngebäude von 39,6\% (1995) auf 68,0\% (2006); in Ostdeutschland lag dieser Wert 2006 sogar bei 83,5\% (BBRWohnungsmarkt-Beobachtungssystem und Statistisches Bundesamt). Im Jahr 2007 fielen die Baugenehmigungen auf einen historischen Tiefstand. Maßgeblich hierfür ist ein drastischer Rückgang bei den Ein- und Zweifamilienhäusern. Wegen der Streichung der Eigenheimzulage zum 1. Januar 2006 zogen viele „Häuslebauer“ ihre Bauaktivitäten vor.

Auch die Baufertigstellungen für Nichtwohngebäude und deren Flächeninanspruchnahme sanken seit Mitte der 1990er Jahre erheblich. Der Wandel der Wirtschaftsstruktur weg von produzierenden und hin zu nutzungsintensiveren Wirtschaftszweigen hat dazu geführt, dass weniger neue Gewerbeflächen ausgewiesen werden. Eine Dienstleistungsgesellschaft verlangt nicht mehr nach großen Produktionsflächen. Der Bedarf der Wirtschaft an Flächen ist aktuell und vermutlich auch auf lange Zeiträume gedeckt (BBR 2007b).
Trotz der Rückgänge im Baubereich blieb die Flächeninanspruchnahme in der letzten Dekade vergleichsweise hoch: Die Gründe liegen u. a. in kleineren Haushalten mit größeren Wohnflächen, der Verlagerung des Wohnungsbaus ganz überwiegend zu Ein- und Zweifamilienhäusern (statt Mehrfamilienhäusern) und der Siedlungstätigkeit in preisgünstigen, eher ländlichen Räumen mit größeren Grundstücken. Vom Zuwachs der Gebäude- und Freiflächen entfallen derzeit bundesweit zwei Drittel auf Wohnbauflächen, der Rest auf Wirtschaftsflächen („Nichtwohnbauflächen“). Mitte der 1990er Jahre betrug das Verhältnis noch etwa 50:50.

Hauptverursacher der Flächeninanspruchnahme sind somit die privaten Haushalte mit ihrem steigenden Bedürfnis nach Wohn- und Erholungsflächen. Die von den privaten Haushalten genutzte Fläche nahm nach der Umweltökonomischen Gesamtrechnung des Statistischen Bundesamtes in den Jahren 1996 bis 2004 um $25 \%$ (+ 69 ha pro Tag) und damit prozentual erheblich stärker zu, als die Zahl der Einwohner im Zeitraum von 1991 bis 2003 $(+2,8 \%)$. Allein der deutlich gestiegene individuelle Wohnflächenkonsum führte $\mathrm{zu}$ einem Anstieg der zum Wohnen genutzten Siedlungsfläche von 1992 bis 2005 um $19 \%$. 


\subsection{Konstanter Zuwachs der Verkehrs- flächen und weitere Fragmentierung}

Im Gegensatz zu den Bauflächen blieb die $\mathrm{Zu}-$ nahme der Verkehrsflächen, darunter ganz überwiegend Wege und Straßen (UBA 2004), mit rund 23 ha pro Tag seit 1993 weitgehend unverändert, nimmt aber in Relation zur rückläufigen Siedlungsflächenzunahme einen größeren Teil der Flächenneuinanspruchnahme ein. Nicht zu vernachlässigen ist dabei auch der landwirtschaftliche Wegebau.

Das Wachstum der Verkehrsflächen führt $\mathrm{zu}$ einer zunehmenden Zerschneidung und Fragmentierung der Landschaft mit negativen Auswirkungen für die vielfältigen Schutz- und Sozialfunktionen (Erhaltung der biologischen Vielfalt, Erholungsfunktion etc.) und ebenso für die land- und forstwirtschaftliche Nutzung. Die Anzahl der unzerschnittenen verkehrsarmen Räume größer als $100 \mathrm{~km}^{2}$ ist in den fünf Jahren von 1998 bis 2003 von 480 auf 422 gesunken, ihre Ausdehnung von $22 \%$ auf $21 \%$ des Bundesgebietes zurückgegangen.

\subsection{Regionale Unterschiede und Trends bei der Siedlungsexpansion}

Unter anderem als Folge der unterschiedlichen demographischen Entwicklung zeichnen sich erhebliche regionale Unterschiede in der Flächenneuinanspruchnahme ab. Berechnungen zeigen, dass das Wachstum zunehmend in Mit- telstädten ${ }^{3}$ und ländlichen Räumen stattfindet sowohl einwohnerbezogen als auch prozentual und absolut (siehe dazu Abb. 2). Zudem entstehen neue Siedlungsgebiete in ihrer Mehrzahl in größerer Distanz zu den Haltepunkten schienengebundener Verkehrsmittel (BBR 2007c). All dies trägt zur Siedlungsdispersion und Zersiedelung bei. Mengenmäßige Zielerreichungsbeiträge für das ,30-ha-Ziel“ lassen sich somit besonders in eher ländlicheren Gemeinden und dem entfernteren Umland der Kernstädte finden, in denen im Jahr 2004 eine überdurchschnittliche Bautätigkeit festzustellen war. Gleichzeitig wird vielerorts das Problem des innerörtlichen Leerstandes und der Brachflächen offenkundig (BBR 2007a), die gerade in ländlich-peripheren Räumen mangels baulicher Nachnutzung ein gravierendes Problem darstellen. Überspitzt könnte man sagen: „Dorfkerne werden den Neubausiedlungen auf der Grünen Wiese geopfert".

Dennoch bleibt Flächenneuinanspruchnahme auch ein Problem in Agglomerationen und wachsenden Regionen, da dort jede zusätzliche Bebauung die wenigen Rest-Freiflächen weiter eingrenzt. Und weil verdichteter gebaut wird, ist die Bodenversiegelung in Agglomerationsräumen deutlich größer als in dünner besiedelten Regionen. In den stark wachsenden Regionen ist die Bautätigkeit in den letzten fünf Jahren signifikant weniger zurückgegangen $(-10 \%)$ als in den schrumpfenden bzw. stark schrumpfenden Regionen (-35\%).

\section{Abb. 2: Regional differenzierte Siedlungs- und Verkehrsflächenzunahme nach 4 Gemeindetypen} (1997-2006, in ha)

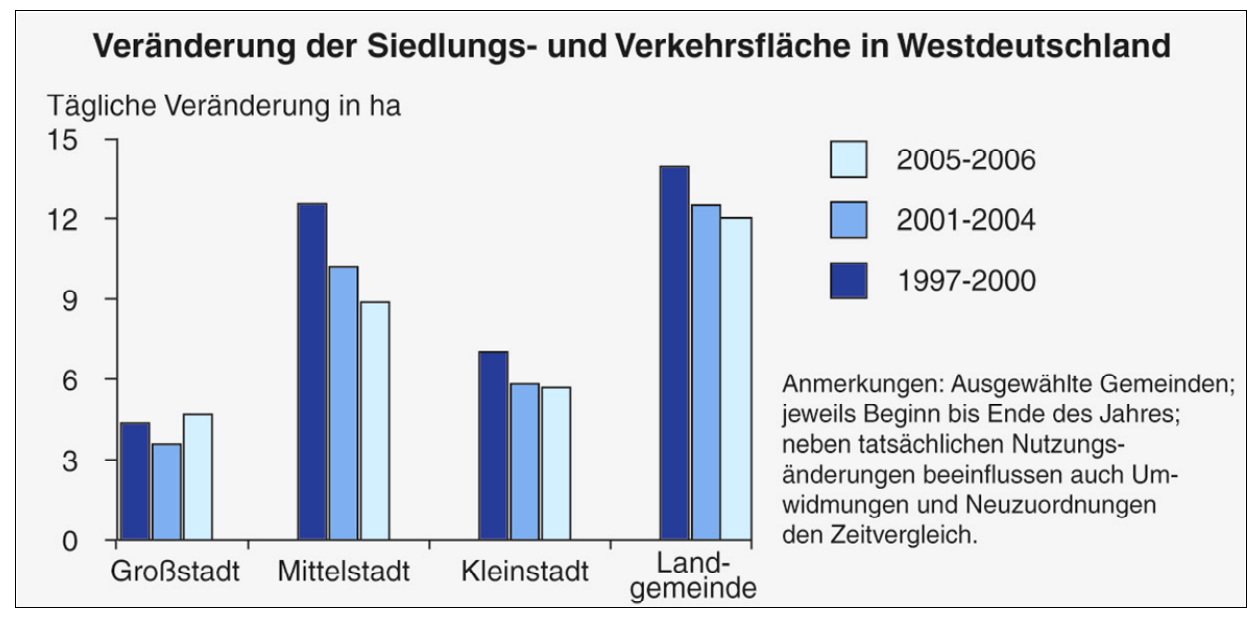

Quellen: Statistische Landesämter, eigene Berechnungen, (C) BBR Bonn 2008 


\subsection{Parallelität von Suburbanisierung und Reurbanisierung}

Die Suburbanisierung war Anfang der 2000er Jahre gegenüber Ende der 1990er Jahre leicht rückläufig - im Osten stärker als im Westen. Gleichzeitig zeichnet sich ein, wenn auch noch nicht statistisch gesicherter Trend zur Reurbanisierung ab. Wachstum und Schrumpfung liegen regional kleinräumig nebeneinander. Die Ursache dafür ist in der demographischen Entwicklung zu finden, welche die Städte aller Regionstypen und alle Stadtteilebenen unabhängig vom innerstädtischen Lagetyp betrifft (DGD 2007).

In den alten Bundesländern fand bereits seit den 1960er Jahren eine Umverteilung von Bevölkerungsanteilen zulasten der Kernstädte statt, die in mehreren Wellen verlief und in den 1990er Jahren einen letzten Höhepunkt erlebte. Das knappe Wohnungsangebot in den westdeutschen Wachstumsräumen führt nach wie vor zur Verdrängung bestimmter Bevölkerungsgruppen an den Stadtrand oder gar - insbesondere in süddeutschen Städten - ins Umland. Allerdings wurden diese Verluste in der Vergangenheit meist durch Binnen- und Außenwanderungsgewinne kompensiert.

Diese stadtregionalen Dekonzentrationsprozesse entfalteten sich in den neuen Bundesländern nach 1990 mit äußerst hoher Dynamik. Seit 2000 hat sich die Suburbanisierung in Ostdeutschland nicht nur drastisch abgeschwächt (Herfert 2007); sie ist sogar weitgehend zum Stillstand gekommen, wie dies auch in altindustrialisierten Regionen im Westen geschah. Nachfrageverschiebungen und interregionale Wanderungsgewinne führen in Ansätzen zu einer „Renaissance der Stadt““.

Von der Gunst des Bevölkerungszuwachses oder zumindest stabiler Entwicklungen werden langfristig aber immer weniger Städte profitieren. Die Tragfähigkeit vieler Mittelund Oberzentren ist daher gefährdet. Ein nachfragebedingter Anstieg der Flächeninanspruchnahme ist also künftig nicht zu erwarten. Nach der Wohnungsmarktprognose des Bundesamtes für Bauwesen und Raumordnung (BBR) ist im Zeitraum 2007 bis 2020 von insgesamt 220.000 Wohneinheiten pro Jahr auszugehen, mit abnehmender Tendenz (BBR 2007a). Der Flächenbedarf der Wirtschaft ist, wie oben schon erwähnt, jenseits regionaler Besonderheiten bundesweit gedeckt. Demgegenüber werden einige wenige Metropolregionen in Deutschland immer stärker wachsen. Hier wird der Nutzungsdruck auf verbliebene Freiräume und stadtnahe Landwirtschaft weiter anhalten.

\subsection{Flächennachfrage für die Bereit- stellung erneuerbarer Energien}

Zusätzliche Flächen werden jedoch nicht nur durch Siedlung und Verkehr beansprucht. Die zunehmende Produktion ,regenerativer“ Energien spiegelt sich auch in einem entsprechenden Flächenverbrauch wider. So gab es Ende 2007 beispielsweise bereits über 19.460 Windkraftanlagen (WKA), bei einem (rückläufigen) Zuwachs von nur noch 883 Anlagen im Jahr 2007. Nach Hochrechnungen des BBR mithilfe der Windparkmethode ${ }^{4}$ wurden im Jahr 2004 ca. 312.000 ha Freiland durch Windkraftanlagen belegt. Dies entspräche etwa $6,8 \%$ der Siedlungs- und Verkehrsfläche im Jahr 2004 oder ca. $50 \%$ des Zuwachses der Siedlungsund Verkehrsfläche im Jahr 2004. ${ }^{5}$ Insbesondere die Kapazitäten der WKAs konzentrieren sich einerseits auf Nord- und Nordostdeutschland sowie andererseits auf den Westen, die Biogasanlagen neben dem Norden und Osten auch auf den äußeren Süden Deutschlands. Durch zunehmendes „Repowering“, d.h. den Ersatz vieler bestehender Anlagenstandorte durch wenige moderne Windräder mit höherer Leistung könnte künftig der Zuwachs von Windkraftanlagen und ihrer Fläche sinken, wenn nicht ein massiver Ausbau realisiert wird.

Nach Jahren des Rückzugs der Landwirtschaft aus der Fläche steigt das Interesse an landwirtschaftlich genutzter Fläche durch den Boom bei der Erzeugung von Bioenergie wieder erheblich. Nach den Ausbauzielen der Bundesregierung (Bundesregierung 2007) soll der Anteil der erneuerbaren Energien im Jahr 2020 für Strom bei $27 \%$, für Wärme bei $14 \%$ und für Kraftstoff bei $17 \%$ liegen. Daraus resultieren erhebliche Flächenansprüche (siehe Kap. 2), die besonders in Agglomerationsräumen zu Konflikten mit der Siedlungsexpansion führen können.

Der Biomasseanbau für energetische Zwecke hat in den vergangenen Jahren kontinuierlich zugenommen und wird vermutlich weiter steigen. Im Aufbau-Ost-Projekt „Kulturland- 
schaftliche Wirkungen eines erweiterten Biomasseanbaus für energetische Zwecke" ermittelte das Leibniz-Zentrum für Agrarlandschaftsforschung (ZALF) entsprechende Flächenansprüche und zeigte beispielhaft in verschiedenen Szenarien auf, wie Biomasse umwelt- und kulturlandschaftsverträglich angebaut werden kann (BBR 2008).

\section{Nutzungskonkurrenzen durch die Siedlungsentwicklung ...}

Die disperse Siedlungsentwicklung führt zu zunehmenden Konkurrenzen um die Flächennutzung. Exemplarisch werden hier drei Typen von Nutzungskonkurrenzen eingehender betrachtet, denen die Siedlungsentwicklung ausgesetzt ist:

\section{$2.1 \quad$... mit Landschafts- und Freiraumschutz}

Mangels Freiflächen ist der Nutzungsdruck auf die stadtnahen Freiflächen in Agglomerationsräumen besonders hoch und damit ist es auch die Konkurrenz zu anderen Nutzungsansprüchen wie Landwirtschaft, Naturschutz und Erholung. Dies gilt z. B. auch für strukturschwache Regionen wie das Ruhrgebiet, in denen trotz geringer Flächenneuinanspruchnahme der Verlust der verbliebenen Freiräume größer ist als die Gewinnung neuer Grün- und Freiflächen aus dem Umbau von Stadtbrachen (Dosch, Porsche 2008, i. E.).

Nach Ergebnissen des Nachhaltigkeitsbarometers Fläche sind die vom Bundesamt für Naturschutz als "schutzwürdig“ eingestuften Landschaften in erheblichem Umfang von Siedlungstätigkeit betroffen (BBR 2007c). Ein Drittel des Zuwachses der Siedlungs- und Verkehrsfläche zwischen 1990 und 2000 erfolgte in schutzwürdigen Landschaften; dabei muss jedoch bedacht werden, dass fast $50 \%$ der Fläche Deutschlands in die Kategorie ,schutzwürdige Landschaft" fallen.

In den 1990er Jahren wurde die fortschreitende Zersiedelung vor allem durch ein geringes $\mathrm{Ma} ß$ an standörtlicher Integration neuer Siedlungsflächen in den Siedlungsbestand vorangetrieben. Nur ein Viertel der Grenzen neuer Siedlungsflächen schließt direkt an den Sied- lungsbestand an. Ebenfalls ein Viertel aller neuen Siedlungsflächen wurde ohne jegliche Angrenzung an den Siedlungsbestand realisiert. Weiterhin ist die Nutzungsintensität neuer Siedlungsflächen regional unterschiedlich und in den nördlichen und östlichen Bundesländern vergleichsweise gering.

Konflikte um Windkraftanlagen können teilweise durch Offshore-Anlagen und Repowering gemindert werden. Über Repowering kann z. B. der „Verspargelung“ der Landschaft entgegengewirkt und die installierte Leistung gesteigert werden. Die erforderlichen neuen Ferntransportleistungen für die neuen Offshore-Windparks verursachen allerdings schon heute auch fernab im Binnenland große Akzeptanzprobleme.

Aus Berechnungen des ZALF zur Bioenergieproduktion wird deutlich, dass die parallele Realisierung der energiepolitischen Ausbauziele der Bundesregierung über die verschiedenen Nutzungsformen von Biomasse (Strom, Wärme und Treibstoff) zu hohen und bundesweit schwer erfüllbaren Flächenansprüchen führt (BBR 2008). Strebt man zudem eine ökologie- und raumverträgliche Ausgestaltung von Biomassepfaden an, so steigt der absolute Flächenbedarf und Transportaufwand. Anlagen zur Gewinnung von Bioenergie stehen derzeit in der Regel auf Standorten außerhalb von Siedlungen und inmitten land- und forstwirtschaftlich genutzter Flächen. Unabhängig vom direkten Flächenverbrauch werden dezentrale Biogas-Kleinanlagen von kommunalen Planungsträgern nach einer Umfrage hinsichtlich Anlagengröße und Landschaftszerschneidung dann als unproblematisch eingeschätzt, wenn es sich um Einzelanlagen handelt und in der Region nur eine begrenzte Anzahl vorhanden ist (BBR 2008). Demgegenüber werden zentrale Biogas-Großanlagen kritischer gesehen, da sich diese insbesondere in der freien Landschaft und in gewachsenen Kulturlandschaften negativ auf das Landschaftsbild auswirken.

\section{$2.2 \quad$... mit stadtwirtschaftlichen Kalkulationen}

Stadtwirtschaftliche Kalkulationen erfordern eine gewisse Mindestauslastung technischer und sozialer Infrastruktur. Siedlungsflächenzunahme kann zu einer Entdichtung des Sied- 
lungsraumes führen und gefährdet besonders in Schrumpfungsräumen die ohnehin vielerorts bereits prekäre Lage der Kommunalhaushalte. Der Pro-Kopf-Aufwand für technische Infrastruktur (z. B. Abwasserentsorgung) ist in Ländern mit geringer Siedlungsdichte signifikant erhöht. Dieser in Deutschland seit Jahrzehnten anhaltende Prozess hat sich in den vergangenen Jahren aufgrund der Abschwächung des Bevölkerungszuwachses verstärkt. Nimmt die Siedlungsdichte weiterhin ab, wächst die Gefahr zunehmender Fragmentierung der Siedlungsstruktur sowie der Unterauslastung von Infrastruktur. Besonders problematisch ist dies bei gleichzeitiger Schrumpfung von Bevölkerung und Infrastruktur, weil dies u. a. mit erheblichen Folgen für die Finanzierung der infrastrukturellen Basisversorgung der Bevölkerung verbunden ist (ARL 2008).

\section{$2.3 \quad$... mit hochwertigen Böden für landwirtschaftliche Nutzung}

Die Dynamik der Siedlungstätigkeit in schutzwürdigen Landschaften ist hoch und die Flächeninanspruchnahme vollzieht sich zu erheblichen Teilen auf Böden mit hoher natürlicher Ertragsfähigkeit, die aber nur $18 \%$ der Gesamtfläche Deutschlands ausmachen (BBR 2007c). Gleichwohl fand ein Drittel der gesamten Flächenneuinanspruchnahme für Siedlungsund Verkehrszwecke im Zeitraum 1996 bis 2000 darauf statt. Dort geht die Option verloren, Nahrungs- und Futtermittel, Energiepflanzen oder nachwachsende Industrierohstoffe anzubauen. Denn fast die Hälfte der Siedlungsund Verkehrsfläche ist versiegelt und dies ist nahezu irreversibel (Gunreben et al. 2007).

\section{Flächenpolitische Bewertung der Siedlungstrends}

Der demographische Wandel, Wanderungsbewegungen und wirtschaftsstrukturelle Veränderungen werden die zwar verminderte, doch anhaltende Neuinanspruchnahme von Flächen für Siedlungen und Verkehr künftig noch stärker in die gesellschaftliche Diskussion rücken. Neben ökologischen Problemen, wie Artenschwund, Zerschneidung und Zerstörung von Biotopen, Beeinträchtigung des Wasserhaushalts und des
Klimas, resultieren aus der Flächenneuinanspruchnahme verstärkt auch ökonomische, baukulturelle und soziale Schwierigkeiten.

\section{1 Überangebot an Bauland}

Verdichtung statt Zersiedelung lautet angesichts dieser Probleme die Devise; sie ist zunehmend auch aus klimapolitischen Erwägungen geboten. Viele Kommunen haben schon heute mit den finanziellen Auswirkungen der Fehleinschätzungen des Baulandbedarfs zu kämpfen. Durch Bevölkerungsrückgang, Alterung, wirtschaftsstrukturelle Verschiebungen und regional unterschiedliche Entwicklungstrends sind bereits deutliche Ausdifferenzierungen auf dem Immobilienmarkt zu verzeichnen. Diese werden sich durch das Überangebot an Bauland - regional unterschiedlich - weiter verschärfen. Leerstand und Siedlungsbrachen sind nicht nur ein Phänomen von Innenstädten, sondern zunehmend auch am Stadtrand und sogar in suburbanen Räumen zu finden. Die zurückgehende Auslastung der technischen und sozialen Infrastruktur ist längst ein Problem für viele Kommunen und Versorger nicht nur in Ostdeutschland. Gleichzeitig wird die Wiedernutzung der immer umfangreicheren Brachenbestände erschwert, obwohl sie letztlich ein zunehmendes städtebauliches Potenzial darstellen.

\subsection{Zunehmend Langfristbrachflächen}

Brachen sind eine notwendige Begleiterscheinung auch in wachsenden, dynamischen Regionen (Berger 2006). Da aber in den 1990er Jahren vielerorts mehr Bauland neu ausgewiesen als angesichts der stagnierenden Bevölkerung und verhaltenen Konjunktur benötigt wurde, nahmen seit dem Jahr 1993 untergenutzte und brachliegende Flächen im Siedlungsbestand deutlich zu. Im Jahr 2006 erfolgte ein Anstieg auf mindestens 150.000 ha; davon befanden sich mehr als 36.000 ha in den neuen Bundesländern und Berlin' .

Prozentual entspricht dies einem Anteil von mehr als $4 \%$ des Bestandes der Siedlungsund Verkehrsflächen im Jahr 2004, mehr als dem Vierfachen der jährlichen Zunahme der Siedlungs- und Verkehrsfläche und mehr als dem Siebenfachen des jährlichen Zuwachses 
der Gebäude- und Freiflächen im Zeitraum 2001 bis 2004.

Das wieder nutzbare Potenzial an Brachflächen im Jahr 2006 wurde nach der Gewerbebaulandumfrage (BBR 2007a) bundesweit auf 63.000 ha hochgerechnet; es entfällt etwa zu gleichen Teilen auf Ost und West und liegt in den ostdeutschen Bundesländern damit prozentual wesentlich höher. Vergleichsweise groß ist die Menge der Siedlungsbrachen in den ostdeutschen und den altindustrialisierten sowie den konversionsbetroffenen Regionen. Bundesweit gibt es Fortschritte bei der Wiedernutzung von Brachflächen. So hat der Anteil der Brachen am neu bereitgestellten Gewerbebauland erheblich zugenommen. Er stieg in einer Vergleichsgruppe der Baulandumfrage von $27 \%(1998 / 99)$ auf $52 \%(2004 / 05)$. Dennoch nehmen seit dem Jahr 2000 die Brachflächen schneller zu als deren Revitalisierung.

Nur bei einem Teil dieser Flächen, vor allem in den heutigen Wachstumsregionen, besteht eine realistische Aussicht auf die baldige
Wiedernutzung als Wohn- oder Gewerbefläche. Im Übrigen können allenfalls Zwischennutzungen realisiert werden (BBR 2004). ${ }^{7}$ Sie dürfen die Kommunen und Eigentümer finanziell allenfalls nur wenig belasten und sollten möglichst zur Verbesserung des Stadtbildes beitragen. Mangels Bauflächennachfrage werden Renaturierung und Zwischennutzungen als Strategie zur Entwicklung von Brachflächen immer wichtiger (Dosch et al. 2008).

Eine Evaluierung der aktuellen Entwicklung vor dem Hintergrund der Ziele einer nachhaltigen Flächennutzung zeigt sowohl zielkonforme wie als problematisch zu bewertende Trends (siehe Tab. 1).

\section{Strategien zur Flächenentwicklung}

Trotz rückläufiger Baufertigstellungen und vielerorts schrumpfender Bevölkerung wird das 30ha-Ziel nur durch eine konsequente Flächeneinsparpolitik erreichbar sein. Auch für den Bürger ist Zersiedelung unter sozialen Aspekten nicht

Tab. 1: Bewertung der Trends zur Flächeninanspruchnahme

\begin{tabular}{|c|c|c|}
\hline Ziel & Zielkonforme Entwicklungen & Problematische Entwicklungen \\
\hline $\begin{array}{l}\text { Reduktion der Flächenneuinan- } \\
\text { spruchnahme }\end{array}$ & $\begin{array}{l}\text { Zunahme neuer Bauflächen deutlich } \\
\text { abgeschwächt }\end{array}$ & $\begin{array}{l}\text { nach wie vor hoher ,Flächen- } \\
\text { verbrauch“; aktuelle Reduktion eher } \\
\text { Folge schleppender Bautätigkeit }\end{array}$ \\
\hline $\begin{array}{l}\text { Erreichung des 30-ha-Ziels bis } \\
\text { zum Jahr } 2020\end{array}$ & $\begin{array}{l}\text { Dynamik des Siedlungsflächenwachs- } \\
\text { tums im Westen auf niedrigstem Niveau } \\
\text { seit } 50 \text { Jahren }\end{array}$ & $\begin{array}{l}\text { zusätzliche Flächeninanspruchnahme } \\
\text { trotz Schrumpfung; abnehmende Sied- } \\
\text { lungsdichte }\end{array}$ \\
\hline Verringerung der Versiegelung & $\begin{array}{l}\text { Versiegelung nimmt langsamer zu als } \\
\text { Flächenverbrauch }\end{array}$ & $\begin{array}{l}\text { Neuversiegelung im Bestand durch } \\
\text { Nachverdichtungen; Neuversiegelung } \\
\text { im Außenbereich durch Anlagen zur } \\
\text { Nutzung erneuerbarer Energien }\end{array}$ \\
\hline Bodenschutz & $\begin{array}{l}\text { Bebauung von Landwirtschaftsflächen } \\
\text { rückläufig }\end{array}$ & $\begin{array}{l}\text { Flächenverbrauch zu einem Drittel auf } \\
\text { hochwertigen Böden }\end{array}$ \\
\hline Ausbau der Bioenergie & $\begin{array}{l}\text { Anbaupotenziale für nachwachsende } \\
\text { Rohstoffe auf Stadtbrachen }\end{array}$ & $\begin{array}{l}\text { Konkurrenz zwischen Nahrungsmittel- } \\
\text { produktion und Bioenergie }\end{array}$ \\
\hline Flächenkreislaufwirtschaft & $\begin{array}{l}\text { Baulandbereitstellung auf Stadtbrachen } \\
\text { nimmt zu; Stadtbrachen als Potenzial für } \\
\text { Zwischennutzungen }\end{array}$ & $\begin{array}{l}\text { Flächenrecycling geringer als Wachs- } \\
\text { tum des Brachenbestandes; Zunahme } \\
\text { von Langfristbrachen }\end{array}$ \\
\hline Innenentwicklung & $\begin{array}{l}\text { Suburbanisierung leicht rückläufig; Reur- } \\
\text { banisierung nimmt zu }\end{array}$ & $\begin{array}{l}\text { Entdichtung des Siedlungsraumes; } \\
\text { steigende Infrastrukturkosten }\end{array}$ \\
\hline Siedlungskonzentration & $\begin{array}{l}\text { Nutzungsintensität neuer Bebauungen in } \\
\text { Teilregionen hoch }\end{array}$ & $\begin{array}{l}\text { Konzentration des Zuwachses auf nicht } \\
\text { zentralörtliche Gemeinden }\end{array}$ \\
\hline Flächenproduktivität & $\begin{array}{l}\text { flächenintensive Produktionsanlagen in } \\
\text { Industrie und Gewerbe nehmen ab }\end{array}$ & $\begin{array}{l}\text { nur ein Fünftel der neuen Siedlungsflä- } \\
\text { chen in fußläufiger Erreichbarkeit zu } \\
\text { Haltepunkten des ÖPNV }\end{array}$ \\
\hline
\end{tabular}

Quelle: Eigene Darstellung 
vertretbar, weil die hohen Kosten falscher Flächenpolitik nicht nur die öffentlichen, sondern über Steuern und Gebühren auch die privaten Haushalte zunehmend belasten.

Zur Reduzierung der Flächenneuinanspruchnahme sind die Wiedernutzung ungenutzter Siedlungsflächen und die maßvolle Nachverdichtung im Bestand unverzichtbar. Wohnungsneubau sollte vorrangig auf Bestands- und Brachflächen im Innenbereich erfolgen. Die Ausweisung neuer Bauflächen ist künftig auf Wachstumsregionen zu konzentrieren. Der strategische Ansatz der Flächenkreislaufwirtschaft und die Ausschöpfung der bestehenden planungsrechtlichen Möglichkeiten stellen dabei ganz entscheidende Grundbedingungen dar.

In der städtebaulichen Praxis ist das Erreichen des 30-ha-Ziels in erster Linie eine Aufgabe der Länder und Gemeinden. Verschiedene Bundesländer haben, insbesondere im Rahmen von „Bündnissen für die Fläche“, zahlreiche Aktivitäten zur Forschung, Förderung und Umsetzung einer nachhaltigen Flächenentwicklung unternommen, und viele Gemeinden widmen sich intensiv dieser Aufgabe. Die Bundesregierung unterstützt dies neben den Förderprogrammen der Städtebauförderung, die auf die Innenentwicklung der Städte und Gemeinden ausgerichtet ist, und den Forschungsprogrammen auch durch die Gesetzgebung.

\subsection{Vieles ist bereits auf Bundesebene initiiert worden}

Der neue §13a BauGB, eingeführt bei der Städtebaurechtsnovelle 2007, wird bereits intensiv von vielen Kommunen genutzt und ermöglicht, für bestimmte Bebauungspläne der Innenentwicklung als rechtlichen Bonus ein vereinfachtes und beschleunigtes Verfahren zu wählen. Im Bereich der Städtebauförderung sind Schwerpunkte u. a. die Stärkung von Innenstädten und Ortsteilzentren, die Wiedernutzung von Brachen sowie von flächensparenden Bauweisen. $\mathrm{Zu}$ den bewährten Städtebauförderungsprogrammen wie Sanierungs- und Entwicklungsmaßnahmen, Stadtumbau Ost und West, Soziale Stadt und Städtebaulicher Denkmalschutz gibt es seit 2008 das neue Programm „Aktive Stadt- und Ortsteilzentren“.

Bereits erfolgt sind die Streichung der Eigenheimzulage zum 1.1.2006, die u. a. aus
Gründen des Abbaus von Fehlsubventionierungen des Wohnungsbaus insbesondere auf der „grünen Wiese“ seit Langem gefordert worden war, sowie die Kürzung der gelegentlich auch als „Zersiedelungsprämie“ bezeichneten Entfernungspauschale. ${ }^{8}$

\subsection{Umsetzungsorientierte Forschungs- programme zur nachhaltigen Sied- lungsflächennutzung}

Forschungsprogramme und Modellvorhaben des Bundes und der Länder fördern die Bestandsentwicklung. Zudem sind zahlreiche Konzepte und Instrumente für eine Mengensteuerung der Flächeninanspruchnahme entwickelt worden.

Im Rahmen des Forschungsfelds „Fläche im Kreis - Kreislaufwirtschaft in der städtischen / stadtregionalen Flächennutzung“" wurden mehr als 50 bestehende und neue Instrumente systematisiert. In fünf Regionen wurden sie in Planspielen auf Zielerreichungsbeitrag, Realisierbarkeit und Akzeptanz getestet. Im Ergebnis ermöglichen schon heute die verfügbaren Instrumente den raschen Einstieg in die Flächenkreislaufwirtschaft (BMVBS, BBR 2007; Dosch 2007); dies geschieht z. B. über integrierte Handlungsrahmen (Dosch et al. 2007).

Zur effektiven und dauerhaften Realisierung einer Flächenkreislaufwirtschaft plädierten die Planspielregionen für einen ,policy mix" aus verbessert eingesetzten verfügbaren Instrumenten und neuen marktwirtschaftlichen Instrumenten. Im Praxistest präferiert wurden u. a. eine Kosten-Nutzen-Betrachtung von Flächenneuausweisungen, Fonds zur Mobilisierung kleinteiliger Brachflächen, zinsbegünstigte Kredite für die Bestandsentwicklung oder eine Baulandausweisungsumlage. Einige dieser Empfehlungen werden in Projekten des BMBF-Förderschwerpunktes REFINA vertieft.

In sieben Modellvorhaben der Raumordnung "Nachhaltige Siedlungsentwicklung“" (2004-2006) standen die Weiterentwicklung integrierter Ansätze des regionalen Flächenmanagements sowie neue ökonomische Anreizinstrumente zum Flächensparen und deren Verknüpfung mit planerischen Verfahren im Mittelpunkt. 
Im Förderschwerpunkt „Forschung für die Reduzierung der Flächeninanspruchnahme und ein nachhaltiges Flächenmanagement" (REFINA) beteiligen sich über 100 Vorhaben in etwa 50 Forschungsverbünden und Einzelprojekten. Sie werden vom BMBF mit rund 22 Mio. Euro gefördert. Dabei bestehen bei den beteiligten Universitäten und Hochschulen, Kommunen, Unternehmen, Verbänden und Ingenieurbüros vielseitige fachliche, methodische und räumliche Zugänge zu Fragen der Reduzierung der Flächeninanspruchnahme und des nachhaltigen Flächenmanagements. REFINA-Vorhaben bearbeiten modellhaft konkrete Einzelstandorte, einzelne Kommunen oder Regionen in allen Bundesländern (BMBF 2008). Dabei werden städtische Räume ebenso berücksichtigt wie ländliche.

\subsection{Perspektivische Maßnahmen}

Lösungswege zur nachhaltigen Siedlungsentwicklung sind seit etlichen Jahren intensiv erforscht, viele handlungsorientierte Ansätze unterbreitet und mit kommunalen und regionalen Praktikern erprobt worden. Da es zunehmend aber nicht mehr nur um das ,Weniger an Flächenverbrauch", sondern vor allem um die Inwertsetzung von Bestandsflächen und das Flächenrecycling geht, bleibt das Thema „Siedlungsflächen“ ein Dauerbrenner nachhaltiger Stadt- und Siedlungsentwicklung. Die derzeit stark steigenden Mobilitätskosten erfordern umso mehr eine kompakte Siedlungsentwicklung. Konkret sind einige Maßnahmen angedacht, die auf einen sparsamen Umgang mit Grund und Boden hinwirken sollen: Dazu zählt die Verankerung einer sparsamen Flächennutzung im Zuge der Novellierung des Raumordnungsgesetzes. Eine Regulierung des Siedlungsflächenwachstums mittels Mengensteuerung durch Regionalpläne wird ebenso verstärkt verfolgt (Einig 2005). Weiterhin soll erforscht werden, wie mithilfe von Grundstückfonds Brachflächen mobilisiert werden können, die von sich heraus nicht marktgängig sind. Zugleich müssen Probleme der Flächeninanspruchnahme transparenter und kalkulierbarer sein, um sie ins Bewusstsein der Menschen rücken zu können. Kosten-Nutzen-Relationen der Siedlungsentwicklung - so eine Erfahrung aus dem Forschungsfeld „Fläche im Kreis“ bieten dazu eine Möglichkeit. In der Forschung könnte es überdies künftig wichtiger werden, verstärkt eine flächensparende, qualitätsvolle und sozialverträgliche Dorf- und Siedlungsentwicklung in ländlichen Räumen zu betrachten, die auch auf eine Nutzung der brachliegenden dörflichen Baubestände ausgerichtet ist und Konkurrenzen und Konflikte mit anderen Nutzungsansprüchen im Blick hat.

Ein relativ neuer Aspekt ist die energetische Nutzung von Stadtbrachflächen z. B. durch den Biomasseanbau (Glöckner 2007). Als Vorteile werden damit die gestalterische und soziale Einbindung in die Umgebung, die Aufhebung der Trennwirkung von Brachflächen, die Steigerung der Attraktivität angrenzender städtebaulicher Entwicklungsflächen durch erhöhten Grünanteil, die Verbesserung der Akzeptanz für diese Nutzungsform sowie die Intensivierung der Auseinandersetzung mit dem Thema Energie in der Bevölkerung verbunden. Als Nachteile gelten u. a. hohe anfängliche Kosten und mögliche Belästigungen durch die Bearbeitung der Fläche sowie durch Ernte und Abtransport der Biomasse (Dosch et al. 2008). Die konkreten Flächenpotenziale sind derzeit gering und eher auf altindustrialisierte Räume mit geringem Baulanddruck, spezifischen Standortpotenzialen ${ }^{9}$ und Wirtschaftlichkeitsbetrachtungen beschränkt. Derzeit werden in vier Regionen Modellvorhaben des BBR / BMVBS im Rahmen des experimentellen Städtebaus durchgeführt.

Negative Effekte eines gesteigerten Anbaus von Biomasse mit ihren zugehörigen Produktionsflächen und neuer Verkehrsinfrastruktur auf die landschaftliche Attraktivität könnten durch die Raumordnung und Raumentwicklung über eine aktive Gestaltung von Energielandschaften gemindert werden. Planerisch abgestimmt, auf Leitbildern basierend und institutionell eingebettet könnten Energielandschaften im Rahmen von „Regionalen Entwicklungskonzepten“ (REK) oder von „Integrierten ländlichen Entwicklungskonzepten“ (ILEK) erarbeitet werden. Sofern Kurzumtriebsplantagen zur Biomasseerzeugung an Bedeutung gewinnen, sollte ein einheitlicher großflächiger Anbau verhindert und stattdessen ihr gestalterisches Potenzial als Strukturierungselement genutzt werden. Möglicherweise könnten „Zonen für Biomasseanlagen“ im Flächennutzungsplan ausgewiesen und damit die 
Privilegierung außerhalb dieser Zonen verhindert werden, um sensible Gebiete zu schützen (BBR 2008).

\section{Anmerkungen}

1) Der Autor dankt Dipl.-Ing. agr. Gisela Beckmann (BBR) für die Durchsicht.

2) Die Siedlungs- und Verkehrsfläche setzt sich aus der Summe mehrerer heterogener siedlungswirtschaftlicher Flächennutzungsarten zusammen: Gebäude- und Freifläche, Betriebsfläche (ohne Abbauland), Erholungsfläche, Verkehrsfläche und Fläche für Friedhöfe. Die Siedlungs- und Verkehrsfläche kann keineswegs mit dem Begriff ,versiegelt“" gleichgesetzt werden, da sie einen nicht quantifizierbaren Anteil von nicht bebauten und nicht versiegelten Frei- und Grünflächen enthält (Statistisches Bundesamt 2007).

3) Mittelstädte sind Städte mit Einwohnerzahlen zwischen 20.000 und 100.000 Einwohnern.

4) Bei der Windparkmethode wird zunächst ein Radius von 500 Metern um jede verortete Windkraftanlage gezogen. Die daraus resultierenden Flächen werden jeweils zu einem Windpark zusammengefasst, wenn sie sich berühren oder überschneiden. Der Kippabstand als Mindestabstand der Anlagen ist nun für alle Windkraftanlagen zu berechnen. Damit der Zuschnitt der Windparks erhalten bleibt, wird die oben ermittelte Fläche nach innen gepuffert (zu Details vgl. Schmitt et al. 2006).

5) Allerdings werden nach derzeitigem Kenntnisstand Standorte für Windkraftanlagen flächenstatistisch nicht als Siedlungs- und Verkehrsfläche erfasst, sondern der land- und forstwirtschaftlichen Nutzung zugeordnet.

6) Das Umweltbundesamt gibt für das Jahr 2004 sogar 176.000 ha ungenutzte Siedlungs- und Verkehrsflächen an (UBA 2008).

7) Erfolgreiche Beispiele für Zwischennutzungen sind u. a. die „Wächterhäuser“ (ein übertragbares Modell für die Sicherung und Zwischennutzung gefährdeter Altbausubstanz, siehe http://www. haushalten-leipzig.de), Parkflächen (wie der Kiezpark Berlin-Prenzlauer Berg) oder die temporäre Nutzung leerer Flächen (z. B. in Dietzenbach, vgl. http://www.werkstatt-stadt.de).

8) Im Gegensatz zu den im Sommer 2008 aufkeimenden Forderungen nach einer Wiedereinführung in der alten Form wäre deren ersatzlose Streichung flächenpolitisch zielführend (vgl. hierzu FOPS-Projekt [FE-Nr. 73.0325/2004] im Auftrag des BMVBS: Wirkungen fiskalischer Steuerungsinstrumente auf Siedlungsstrukturen und Personenverkehr vor dem Hintergrund der Nach- haltigkeitsziele der Bundesregierung. http://www. bbr.bund.de/cln_005/nn_28646/DE/Forschungspr ogramme/FOPS/Projekte/FiskalischeInstrumente/ 03 Ergebnisse.html, download 6.9.08).

9) Vgl. dazu die Website des BBR mit Zwischenergebnissen zur „Nutzung städtischer Freiflächen für erneuerbare Energien“" (http://www.bbr.bund. de/DE/ForschenBeraten/Fachpolitiken/Energie Umwelt/RegenerativeEnergie/ReFoProjekte/ ReFoProjekte.html, download 3.9.08)

\section{Literatur}

ARL - Akademie für Raumforschung und Landesplanung, 2008: Aus der Kostenfalle hin zu mehr Kostenwahrheit: Kosten und Folgekosten von Siedlungen und Infrastrukturen. Positionspapier Nr. 76, Hannover

Berger, A., 2006: Drosscape. Wasting Land in Urban America. New York

BBR - Bundesamt für Bauwesen und Raumordnung (Hg.), 2004: Zwischennutzung und neue Freiflächen - Städtische Lebensräume der Zukunft. Sonderveröffentlichung, Bonn

BBR - Bundesamt für Bauwesen und Raumordnung, 2006: Gewerbeflächenmonitoring. Ein Ansatz zur Steigerung der Wettbewerbsfähigkeit des Gewerbeflächenpotenzials in Ostdeutschland. Forschungen, Heft 119, Bonn

BBR - Bundesamt für Bauwesen und Raumordnung, 2007a: Wohnungs- und Immobilienmärkte in Deutschland 2006. Berichte, Bd. 27, Bonn

BBR - Bundesamt für Bauwesen und Raumordnung, 2007b: Regionale Siedlungsflächenentwicklung in den neuen Bundesländern auf Basis von Prognosen der Bau- und Immobilienwirtschaft. Forschungen, Heft 123, Bonn

BBR - Bundesamt für Bauwesen und Raumordnung, 2007c: Nachhaltigkeitsbarometer Fläche - Regionale Schlüsselindikatoren nachhaltiger Flächennutzung für die Fortschrittsberichte der Bundesregierung. Forschungen, Heft 130, Bonn

BBR - Bundesamt für Bauwesen und Raumordnung (Hg.), 2008: Grünes Gold im Osten?! Flächenansprüche von Biomassepfaden durch klimabedingte Ausbauziele und Handlungsoptionen für die Raumordnung. Bearb. Uckert, G. et al., Leibniz-Zentrum für Agrarlandschaftsforschung (ZALF) e.V. in Zusammenarbeit mit Büro hochC Landschaftsarchitektur, Bonn, Müncheberg; http://lis4.zalf.de/publ/publ1/ alle.htm (download 8.9.08)

BMBF - Bundesministerium für Bildung und Forschung (Hg.), 2008: Wege zum nachhaltigen Flächenmanagement - Themen und Projekte des Förderschwerpunkts REFINA. Bearb. Deutsches Insti- 
tut für Urbanistik, Berlin, $66 \mathrm{~S}$; ; http://www.refinainfo.de/de/refina-veroeffentlichungen/index.phtml (download 8.9.08)

BMVBS; BBR - Bundesministerium für Verkehr, Bau und Stadtentwicklung; Bundesamt für Bauwesen und Raumordnung (Hg.), 2007: Kreislaufwirtschaft in der städtischen / stadtregionalen Flächennutzung. Werkstatt: Praxis 51, Bonn

Bundesregierung 2007: Eckpunkte für ein integriertes Energie- und Klimaprogramm. Meseberg, Berlin DGD - Deutsche Gesellschaft für Demographie e.V., 2007: Städte im demographischen Wandel. Diskussionspapier, Wiesbaden

Dosch, F., 2007: Flächenkreislaufwirtschaft in Regionen des Stadtumbaus - Forschungsaktivitäten des BBR und BMBVS. In: Genske, D.; Ruff, A. (Hg.): Nachhaltiges Flächenmanagement - Potenziale und Handlungsfelder für Regionen im Umbau. Nordhausen, S. 41-55

Dosch, F.; Preuss, T.; Jakubowski, P. et al., 2007: Flächenkreislaufwirtschaft als Strategie zur Steuerung der Siedlungsentwicklung im Bund und in Thüringen. LKV 3-2007. In: Landes- und Kommunalverwaltung. Verwaltungsrechts-Zeitschrift für die Länder Berlin, Brandenburg, MecklenburgVorpommern, Sachsen, Sachsen-Anhalt und Thüringen. 17/9 (2007), S. 385-390

Dosch, F.; Porsche, L., 2008 / i. E.: Grüne Potenziale unter blauem Himmel. Neue Zugänge zur Flächenrevitalisierung und Freiraumentwicklung im Ruhrgebiet. In: Informationen zur Raumentwicklung Heft 9/2008, Bonn

Einig, K., 2005: Regulierung des Siedlungsflächenwachstums als Herausforderung des Raumordnungsrechts. In: DISP 41/160 (2005), S. 48-57

Glöckner, S., 2007: Biomasse im Kurzumtrieb. Entwicklungspotenzial für Altstandorte in dicht besiedelten Regionen. Während ExWoSt-Auftaktveranstaltung „Potenziale städtischer Freiflächen zur Erzeugung erneuerbarer Energien“. http://www.fh-nord hausen.de/uploads/media/9_gloeckner.pdf (download 3.9.08)

Gunreben, M.; Dahlmann, I.; Frie, B. et al., 2007: Die Erhebung eines bundesweiten Indikators Bodenversiegelung. In: Bodenschutz 2 (2007), S.34-38

Herfert, G., 2007: Regionale Polarisierung der demographischen Entwicklung in Ostdeutschland - Gleichwertigkeit der Lebensverhältnisse? In: Raumforschung und Raumordnung 65/5 (2007), S. 435-455

RNE - Rat für nachhaltige Entwicklung, 2008: Welche Ampeln stehen auf Rot? Stand der 21 Indikatoren der nationalen Nachhaltigkeitsstrategie auf der Grundlage des Indikatorenberichts 2006 des
Statistischen Bundesamtes. Stellungnahme des Rates für Nachhaltige Entwicklung, Berlin

Schmitt, M.; Dosch, F.; Bergmann, E., 2006: Flächeninanspruchnahme durch Windkraftanlagen. Berichte aus Forschung und Praxis. In: Raumforschung und Raumordnung 64/5 (2006), S. 405-412

Statistisches Bundesamt, 2007: Flächenerhebung nach Art der tatsächlichen Nutzung. Wiesbaden

UBA - Umweltbundesamt, 2004: Reduzierung der Flächeninanspruchnahme durch Siedlung0 und Verkehr - Materialienband. UBA Texte 90/03, Berlin

UBA - Umweltbundesamt, 2008: Schutz der biologischen Vielfalt und Schonung von Ressourcen Warum wir mit Flächen sorgsam und intelligent umgehen müssen. Dessau, $23 \mathrm{~S}$.

ZDB - Zentralverband Deutsches Baugewerbe, 2008: Baumarkt 2007. Berlin

\section{Kontakt}

Dr. Fabian Dosch

Bundesamt für Bauwesen und Raumordnung (BBR)

I 5 - Umwelt und Verkehr

Deichmanns Aue 31-37, 53179 Bonn

Tel.: +49 (0) 228 / 99 - 401 - 2307

E-Mail: fabian.dosch@bbr.bund.de 